\title{
A Machine Learning-Based Intelligence Approach for Multiple-Input/Multiple-Output Routing in Wireless Sensor Networks
}

\author{
V. Sridhar, ${ }^{1}$ K. V. Ranga Rao, ${ }^{2}$ V. Vinay Kumar, ${ }^{3}$ Muaadh Mukred $\mathbb{D}^{,},{ }^{4}$ Syed Sajid Ullah, ${ }^{5}$ \\ and Hussain AlSalman ${ }^{6}$ \\ ${ }^{1}$ Department of ECE, Vidya Jyothi Institute of Technology, Hyderabad, Telangana, India \\ ${ }^{2}$ Department of CSE, Neil Gogte Institute of Technology, Hyderabad, Telangana, India \\ ${ }^{3}$ Department of ECE, Anurag University, Venkatapur, Telangana, India \\ ${ }^{4}$ Sana'a Community College, Mareb Street, Al-Hushaishiya Road, Sana'a, Yemen \\ ${ }^{5}$ Department of Information and Communication Technology, University of Agder, Kristiansand, Norway \\ ${ }^{6}$ Department of Computer Science, King Saud University, Riyadh 11543, Saudi Arabia \\ Correspondence should be addressed to Muaadh Mukred; muaadh@scc.edu.ye
}

Received 27 November 2021; Revised 22 December 2021; Accepted 3 January 2022; Published 24 January 2022

Academic Editor: Ewa Rak

Copyright (c) 2022 V. Sridhar et al. This is an open access article distributed under the Creative Commons Attribution License, which permits unrestricted use, distribution, and reproduction in any medium, provided the original work is properly cited.

\begin{abstract}
Computational intelligence methods play an important role for supporting smart networks operations, optimization, and management. In wireless sensor networks (WSNs), increasing the number of nodes has a need for transferring large volume of data to remote nodes without any loss. These large amounts of data transmission might lead to exceeding the capacity of WSNs, which results in congestion, latency, and packet loss. Congestion in WSNs not only results in information loss but also burns a significant amount of energy. To tackle this issue, a practical computational intelligence approach for optimizing data transmission while decreasing latency is necessary. In this article, a Softmax-Regressed-Tanimoto-Reweight-Boost-Classification(SRTRBC-) based machine learning technique is proposed for effective routing in WSNs. It can route packets around busy locations by selecting nodes with higher energy and lower load. The proposed SRTRBC technique is composed of two steps: route path construction and congestion-aware MIMO routing. Prior to constructing the route path, the residual energy of the node is determined. After that, the residual energy level is analyzed using softmax regression to determine whether or not the node is energy efficient. The energy-efficient nodes are located, and numerous paths between the source and sink nodes are established using route request and route reply. Following that, the SRTRBC technique is used for congestion-aware routing based on buffer space and bandwidth capability. The path that requires the least buffer space and has the highest bandwidth capacity is picked as the optimal route path among multiple paths. Finally, congestion-aware data transmission is used to minimize latency and data loss along the route path. The simulation considers a variety of performance metrics, including energy consumption, data delivery rate, data loss rate, throughput, and delay, in relation to the amount of data packets and sensor nodes.
\end{abstract}

\section{Introduction}

Wireless sensor networks (WSNs) help in monitoring and recording the physical environmental conditions as well as transmitting the collected data to base station. The networkon-chip (NoC) architecture was constructed in [1] to perform the efficient communication between the multiple cores. The designed system performed on-chip antennas utilization between long distance cores to reduce the latency. However, energy efficiency was not improved by networkon-chip (NoC) architecture.

An opportunistic routing was carried out in [2] for MIMO in wireless ad-hoc networks (WANETs). But, the latency was not reduced by opportunistic routing. A new congestion-aware routing algorithm was introduced in [3] that splits the network-on-chip (NoC) into number of 
subnets. But the computational complexity was not reduced by congestion routing aware algorithm.

A new functional gradient boosting was introduced in [4] for learning the deep residual networks on multiclass classification tasks. However, the packer delivery ratio was not reduced by functional gradient boosting. Two boosting algorithms termed RBoost1 and RBoost2 were introduced in [5], robust to noisy data with AdaBoost. RBoost1 and RBoost 2 failed to focus on samples misclassified by base learners.

A congestion-aware routing algorithm was introduced in [6] through sending the congestion information in data packet. Congestion Aware Routing using Fuzzy Rule sets (CARF) was designed in [7] for managing the excess traffic conditions through finding the nonlocalized node paths. But throughput level was not improved by CARF.

A routing method was designed in [8] to reduce the congestion. The weights estimated from congestion-related parameters were sent to subsequent modules, but the routing cost was not minimized by designed method. Congestion Aware Lifetime Improving Routing Protocol (CALIRP) was designed in [9] to increase the network lifetime through reducing the congestion. However, the computational complexity was not reduced by CALIRP. A congestion-aware clustering and routing (CCR) protocol was designed in [10] to avoid the congestion problem over network. However, the data delivery rate was not improved by designed CCR protocol.

The issues identified from the above literature are higher computational cost, lesser energy efficiency, lesser network lifetime, lesser data delivery rate, lesser computational complexity, higher packet loss rate, higher latency, etc. In order to address these problems, a congestion-aware routing technique called Softmax Regressed Tanimoto Reweight Boost Classification (SRTRBC) method for reliable and congestion routing aware in WSNs is designed.

The main contributions of the work are as follows:

(i) A machine learning concept that combines the weak learners to form the strong one is used for developing the proposed SRTRBC method. The main aim of SRTRBC method is to route the packets around congested areas through identifying the energy-efficient and underloaded nodes. SRTRBC method comprises two processes like route path construction and congestion aware MIMO routing. The residual energy of node is determined for route path construction.

(ii) Softmax regression analysis examined the residual energy level to identify if the node is energy efficient node or nonenergy efficient node. The energy-efficient nodes are identified and constructed through the multiple paths from source node to sink node with help of route request and route reply.

(iii) Tanimoto Reweight Boost Classification is carried out for congestion aware routing depending on buffer space and bandwidth capacity. The route path with lesser buffer space and higher bandwidth capacity is selected as an optimal route path among multiple paths. At last, congestion-aware data delivery is performed along route path to minimize the latency and packet loss.

The rest of the paper is structured as follows: Section 2 reviews the related works of congestion routing aware in WSNs. Section 3 provides brief explanation of the proposed SRTRBC method with neat diagram. In Section 4, simulation settings and result analysis are discussed. The conclusion is given in Section 5.

\section{Related Works}

A congestion control algorithm depending on multiobjective optimization algorithm called PSOGSA was introduced in [11] for rate optimization. The network lifetime was not reduced by congestion control algorithm. A reliable and congestion based protocol was introduced in [12] for enhancing the reliability results. The designed protocol used Order Preference by Similarity to Ideal Solution (TOPSIS) technique to choose an optimal path for data transmission. However, the computational cost was not minimized by reliable and congestion based protocol.

A centralized congestion control routing protocol depending on multimetrics (CCRPM) was introduced in [13] to minimize the network congestion effects. The computational complexity was not reduced by CCRPM. An Enhanced Congestion Control-Random Early Detection (EnCoCo-RED) was designed in [14] to enhance the congestion control performance. Though the congestion control was carried out, the energy efficiency was not improved by EnCoCo-RED.

A fuzzy sliding mode congestion control algorithm (FSMC) was designed in [15] for WSN. However, the packet loss rate was not reduced by FSMC. A congestion control mechanism was designed in [16] on optimized rate for energy efficient transmissions. Though the energy efficiency was improved, routing overhead was not reduced by congestion control mechanism.

An efficient congestion avoidance approach was introduce in [17] with Huffman coding algorithm to enhance the network performance. An ant colony optimization was used to identify the congestion-free alternate paths. However, the complexity level was not reduced by congestion avoidance approach.

A dynamic approach was designed in [18] for queue management to identify the congestion with minimal time consumption and prevent the collision. The back-pressure method was employed depending on queue quality to minimize the linking probability in pathway. The latency was not reduced by dynamic approach.

A PACC protocol was designed in [19] for improving the QoS performance in WSNs, namely latency, data delivery ratio, loss ratio, and network channel efficiency. Though the 
data delivery rate was improved, the time consumption was not reduced by PACC protocol.

A two-level Fuzzy-Logic-Controller- (FLC-) based dynamic clustering scheme was designed in [20] for congestion prevention. The sink nodes forecasted the current load depending on the loads in preceding rounds, but the clustering accuracy was not improved by FLC-based dynamic clustering scheme.

\section{Methodology}

WSN is a group of sensor nodes to transfer the sensed data to sink node. Multiple-input/multiple-out (MIMO) technology is wireless communication used for transmitting the multiple data signals. MIMO method plays an essential role in 3G, 4G, and 4G LTE networks. WSN has experienced from different problems like frequently varying the network topology and congestion problems which affect network bandwidth usage. Congestion occurs when resource demand increase the system available capacity to reduce the latency and packet loss. The congestion avoidance is equal to the traffic load balancing in WSN. The possible congestion avoidance affects the excessive traffic distribution. Consequently, a new scheme is required to enhance the data delivery and minimize the latency. In this paper, reliable and congestion-aware routing technique called SRTRBC method is designed to route the packets around the congested areas by identifying the energy-efficient and underloaded nodes. The key idea of SRTRBC method is to construct the energyefficient path identification and congestion aware routing correspondingly.

Figure 1 reveals the structural design of proposed SRTRBC method to perform the route path identification and MIMO routing in wireless technology. The proposed method computes the energy for every distributed sensor node. After that, the route path between the source and destination is identified through transmitting the request and reply message. The route with minimum distance is selected for efficient routing. In data transmission, the congestion-aware routing is carried out to minimize the network congestion with minimal latency.

3.1. Network Model. A system model of proposed SRTRBC method is introduced. The number of sensor nodes ' $S N_{i}=S N_{1}, S N_{2}, S N_{3} \ldots S N_{n}$ ' are distributed in the squared area ' $n * n$ ' within the transmission range ' $T R$ '. The sensor nodes are located independently and collect the information from environment. The collected data packets ' $\mathrm{d} p_{j}=\mathrm{d} p_{1}, \mathrm{~d} p_{2}, \ldots, \mathrm{d} p_{m}$ ' are sent to the destination node ' $d n$ ' through energy-efficient and congestion aware intermediate nodes ' $I_{1}, I_{2}, \ldots, I_{n}$ ' to improve the lifetime in WSN and to choose the route paths for avoiding the congestion.
3.2. Softmax Regression Analysis-Based Route Path Construction. The SRTRBC method performs the route path classification between sensor nodes through performing the softmax regression analysis. The softmax regression analysis is a machine learning technique to find the relationship between the dependent variable (i.e., 'outcomes') and independent variables (i.e., features). The softmax regression function observes the sensor node features (i.e., energy). For every sensor node, energy level is calculated. Initially, all sensor nodes have similar energy level and it gets minimized during the sensing process. The energy level is computed as product of power and time. It is determined as

$$
\text { Energy }_{S N}=\text { Power } * \text { Time. }
$$

From (1), 'Energy ${ }_{S N}$ ' represents the energy level of sensor nodes. 'Power' is measured in terms of watts. The time is measured in terms of seconds (sec). The energy of sensor node is computed in terms of joule $(\mathrm{J})$. The energy level of sensor gets reduced during the sensing process in wireless network. Consequently, the residual energy of node is determined as

$$
\text { Residual }_{\text {Energy }_{S N}}=\text { Total }_{\text {Energy }_{S N}}-\text { Consumed }_{\text {Energy }_{S N}} .
$$

From (2), 'Residual Energy $_{S N}$ ' represents the residual energy of the sensor node, 'Total Energy $_{S N}$ ' represents the total energy of sensor nodes, and 'Consumed Energy $_{S N}$ ' denotes the consumed energy. The softmax regression analysis in SRTRBC method examines the predicted node energy level and finds the energy-efficient nodes. Softmax regression analysis is the simplification of logistic function to multiple dimensions. It is employed as the activation function to categorize the sensor node output to the probability distribution over the predicted output classes. It is formulated as

$$
S R A=\frac{e^{z_{i}}}{\sum_{k=1}^{p} e^{z_{k}}}
$$

From (3), ' $\sigma(z)$ ' denotes the softmax regression analysis. ' $z_{i}$ ' represents the residual energy of the sensor node ' $i$ ' ranges from 0 to $p$. Normally, the output of the softmax regression analysis ranges from ' 0 to 1 '. When regression analysis provides the output ranges from ' 0 to 0.5 ', then the sensor node is said to nonenergy efficient node. When the regression analysis provides the output ranges from ' 0.5 to 1 ', then the sensor node is said to be energy efficient node. After performing the regression analysis, the node with higher residual energy is chosen for route path construction. The number of route paths is constructed between the source node and destination node using two control messages like route request ' $R_{\text {req }}$ ' and route reply ' $R_{\text {req }}$ '. The source node distributes the request message to the destination node through the intermediate nodes.

$$
\text { Source node } \stackrel{R_{\text {req }}}{\Rightarrow} \sum_{k=1}^{d}\left(\text { Intermediate } \text { nodes }_{k}\right) \stackrel{R_{\text {req }}}{\Rightarrow} \text { Destination Node. }
$$




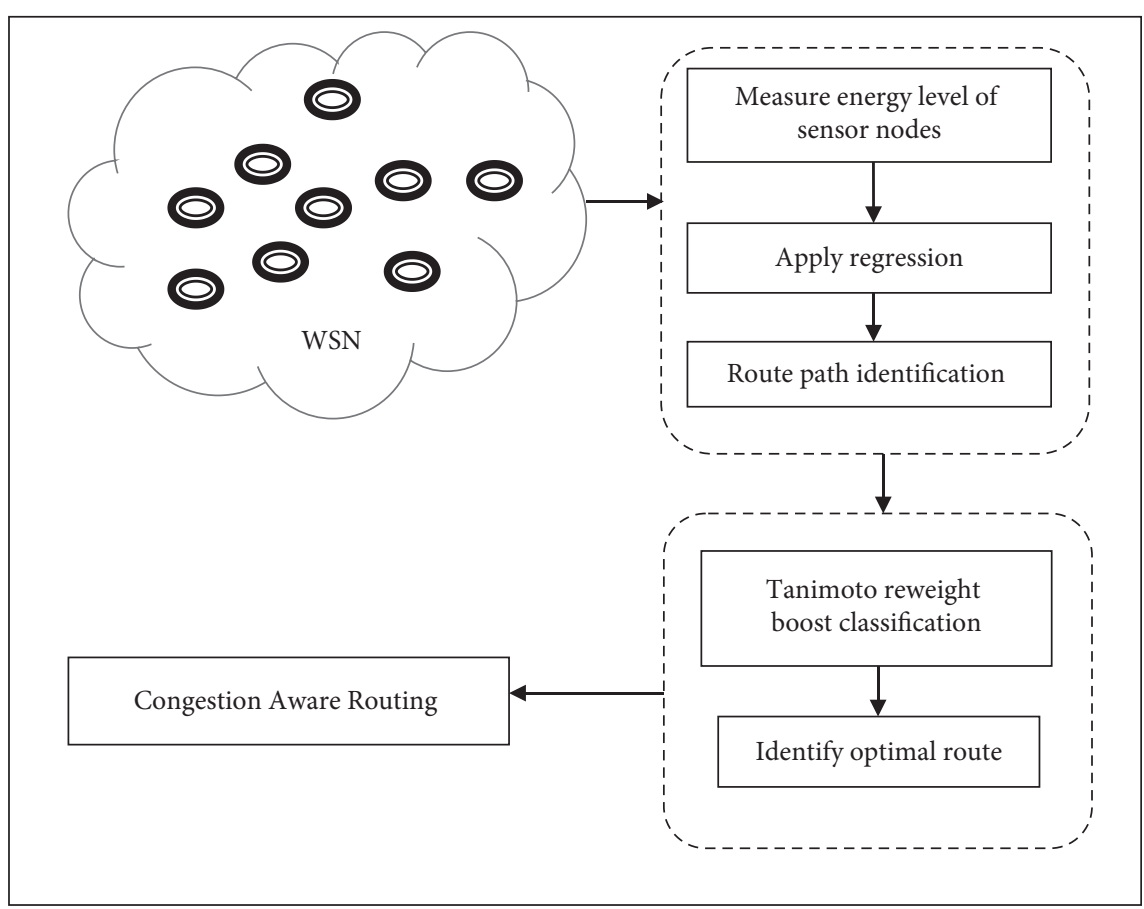

FIGURE 1: Structural design of the proposed SRTRBC method.

The source node transmits the route request packet to the sink node through the intermediate nodes. After collecting the request message, the destination node replies to the source node.

Source node $\stackrel{R_{\text {rep }}}{\models} \sum_{k=1}^{d}\left(\right.$ Intermediate nodes $\left._{k}\right) \stackrel{R_{\text {rep }}}{\models}$ Destination Node.

From (5), ' $R_{\text {req }}$ ' denotes the reply from destination node ' $d n$ ' to the source node ' $s n$ ' via the intermediate nodes $\left(I_{i}\right)$. Depending on two control message transmission, the multiple routes from source to the destination are illustrated in Figure 2.

Figure 2 demonstrates the multiple paths between source and destination node in SRTRBC method. The route paths from above graph are listed in Table 1.

Table 1 describes the multiple route paths from source to destination node. There are three route paths ' $r_{1}, r_{2}$ and $r_{3}$ ' given in Table 1 . The third column represents the number of hops between the source and destination. The multiple route paths are constructed in SRTRBC method from source to destination with higher network lifetime.
3.3. Reweight Adaptive Boosting Classification. Boosting is a machine learning algorithm that combines the weak learners to form the strong one. Boosting classifier is a strong classifier that presents the accurate classification results through combing all the weak learners. As a result, SRTRBC method employs the reweight adaptive boosting classification algorithm to increase the classification performance. The boosting algorithm uses the Tanimoto similarity function in SRTRBC method to categorize the route path into congestion aware and noncongestion aware route path. The flow process of reweight adaptive boosting classifier is illustrated in Figure 3.

Figure 3 shows the structure of the reweight adaptive boost ensemble classifier in SRTRBC method to classify the route path into different classes for further processing. Let us consider the training data as multiple route paths ' $r_{1}, r_{2}$ and $r_{3}$ ' and ' $y_{1}, y_{2}$ and $y_{3}$ ' represent the classification output. The set of weak learners $\left\{w_{1}, w_{2}, w_{3} \ldots w_{n}\right\}$ are constructed to train the input and combined to form the strong one. A decision stump is the decision tree where route path is classified based on the similarity measure. Tanimoto similarity correlation is used in SRTRBC method to determine the linear relationship between congestion-free route path and constructed route path. It is measured as follows:

TSCC $=\frac{\mid \text { congestion }- \text { free route path } \cap \text { constructed route path } \mid}{\mid \text { congestion }- \text { free route path }|+| \text { constructed route path }|-| \text { congestion }- \text { free route path } \cap \text { constructed route path } \mid}$ 


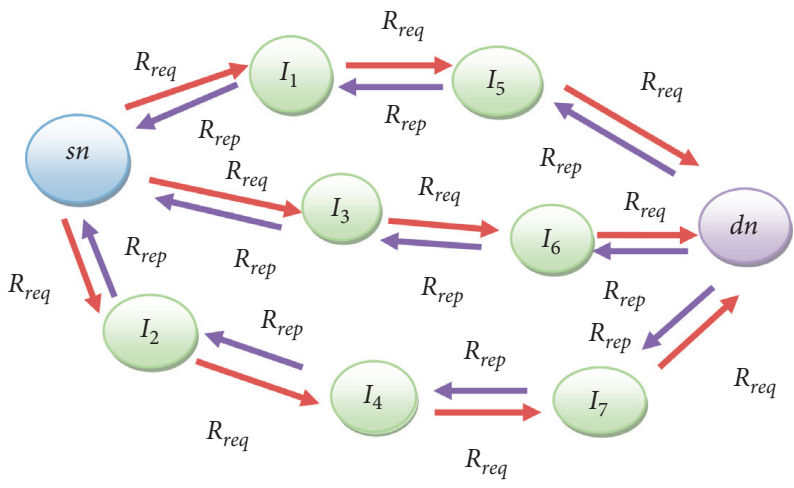

FIgURE 2: Route path construction.

TABLE 1: Tabulation of route paths.

\begin{tabular}{lcc}
\hline Route & Route paths & Hops \\
\hline$r_{1}$ & $s n \longrightarrow I_{1} \longrightarrow I_{5} \longrightarrow d n$ & 2 \\
$r_{2}$ & $s n \longrightarrow I_{3} \longrightarrow I_{6} \longrightarrow d n$ & 2 \\
$r_{3}$ & $s n \longrightarrow I_{2} \stackrel{I_{4}}{\longrightarrow} I_{7} \longrightarrow d n$ & 3 \\
\hline
\end{tabular}

From (6), the Tanimoto similarity correlation coefficient 'TSCC' provides the value between 0 and 1 . The threshold value of softmax regression analysis is set through taking the average of maximum and minimum value. The minimum value is considered as zero and the maximum value is considered as 1 . Through taking the average value, we obtain the threshold value as 0.5 . If the Tanimoto similarity correlation value ranges from ' 0 to 0.5 ', it denotes noncongestion aware path. If the Tanimoto similarity correlation value ranges from ' 0.5 to 1 ', it denotes congestion aware path. Based on the correlation value, the decision dump categorizes the route path into two different classes. After classification, the weak learners are joined into forming the strong learner. It is mathematically formulated as

$$
W C=\sum_{j=1}^{l} w_{j} .
$$

From (7), ' $W C$ ' denotes the output of weak classifier and ' $w_{j}$ ' symbolizes the number of weak learner output. After that, the similar weight is allocated in SRTRBC method to each weak learner. It is calculated as

$$
W C=\sum_{j=1}^{l} \delta * w_{j} .
$$

From (8), ' $\delta$ 'represents the similar weight allocated to the weak learners ' $w_{j}$ '. After that, the training error of each base learner is computed as

$$
\text { Error }=\left(y_{j}-w_{j}\right)^{2} .
$$

From (9), ' $y_{i}$ ' represents the actual output and ' $w_{j}$ ' symbolizes the observed output. Depending on the error, every base learner is reweighted. The boosting classifier identifies the classification results with lesser error rate. The reweighted classification results are attained as

$$
\mathrm{RCR}=\sum \delta^{w} * w_{j}
$$

From (10), 'RCR' represents the output of reweighted classification results. ' $\delta$ ' denotes the reweight of weak learner ' $w_{j}$ '. Depending on the classification results, the route path is correctly classified into two classes. After that, source node sends the data packets to the destination node in congestion-free manner for further processing. The algorithmic process of reweight adaptive boosting classification in SRTRBC method is described as follows.

Algorithm 1 explains the softmax regressed Tanimoto reweight boost classification based reliable and congestion aware routing process. For every sensor node in WSN, the residual energy level is determined. Then, the regression function examines the estimated energy level of sensor node. Depending on regression analysis, the energy-efficient nodes are selected for MIMO routing. With help of energy-efficient nodes, multiple route paths are constructed between source and destination node. The multiple route paths are recognized through transmitting two control messages like route request and reply from source to the destination. The ensemble technique constructs the number of weak learners. After that, the weak learners are combined and similar weight is allocated to the weak learners. Subsequently, the error is determined for each classification result of the weak learner to improve the classification performance. After that, the weak learner weights are recomputed consistent with the error value. Afterwards, the classifier with minimum error is chosen as final strong classification results. Finally, the congestion-free data transmission is carried out in efficient manner with higher data delivery.

\section{Simulation Settings and Result Discussion}

The simulation of SRTRBC method and existing methods is done using MATLAB. In order to conduct the simulation, 1000 sensor nodes are considered as input. The proposed and existing method performance is determined with help of ten runs. The performance analysis of the proposed SRTRBC method and existing methods namely network-on-chip (NoC) architecture [1] and opportunistic routing [2] are explained with five different metrics, namely energy efficiency, data delivery rate, data loss rate, throughput, and latency. The performances of different techniques are 


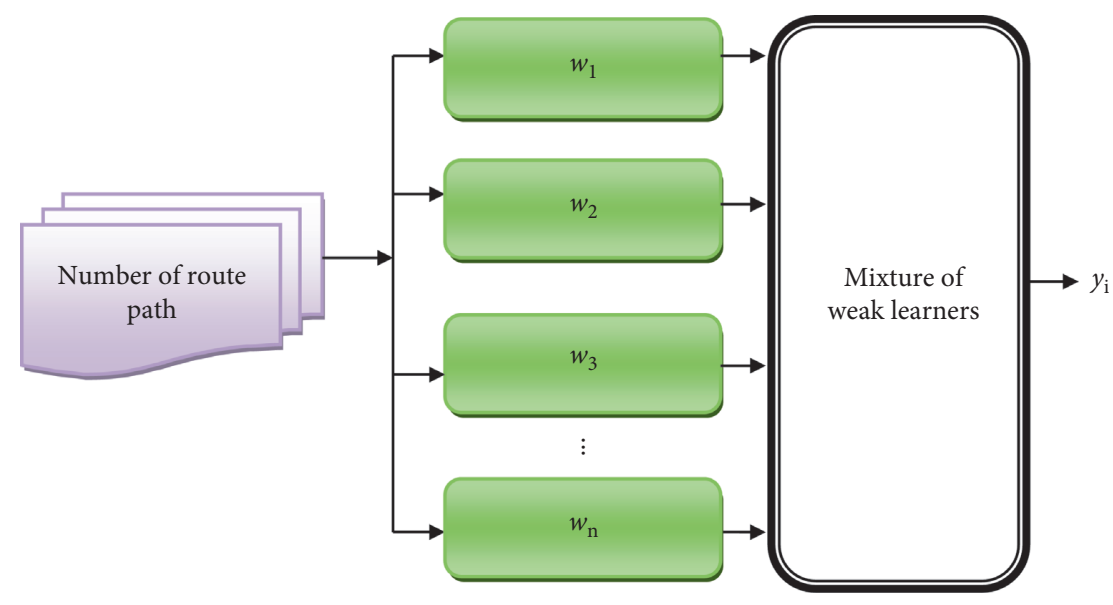

Figure 3: Structure of reweight adaptive boost classification.

Input: sensor Nodes $S N_{1}, S N_{2}, S N_{3} \ldots S N_{n}$, source node, destination node Output: improve congestion aware routing in WSN

Begin

(1) For each $S N_{i}$

(2) Measure residual energy of sensor node

(3) Analyze the residual energy by using softmax regression

(4) if $(S R A<0.5)$, then

(5) $S N_{i}$ is energy-efficient node

(6) else

(7) $S N_{i}$ is nonenergy efficient node

(8) end if

(9) end for

(10) Create the route paths between source and destination node

(11) $s n$ sends $\operatorname{Req}_{R}$ to sink

(12) $s n$ send $\operatorname{Rep}_{R}$ to source

(13) Construct the multiple routes ' $r_{1}, r_{2}$ and $r_{3}$ '

(14) for each constructed route path

(15) Construct ' $n$ ' weak learners

(16) Measure the Tanimoto similarity correlation

(17) Classifies route path into two different classes

(18) Combines all weak learners $y_{i}$

(19) Assign similar weights to the weak classifier

(20) Compute the error

(21) Update the weight of weak classifiers

(22) Find classifier with minimum error

(23) Attain the strong classification results

(24) End for

End

Algorithm 1: Softmax regressed Tanimoto reweight boost classification-based reliable and congestion aware routing algorithm.

analyzed with help of table and graphical illustration. The parameters are explained as given below.

4.1. Impact of Energy Efficiency. Energy efficiency is defined as the proportion of output energy and input energy. Energy efficiency is determined as

$$
\text { Ener }_{\text {Eff }}=\frac{O_{\text {energy }}}{I_{\text {energy }}} \times 100 .
$$

From (11), 'Ener ${ }_{\text {Eff }}$ 'denotes the energy efficiency. Energy efficiency is computed in terms of percentage (\%). Table 2 describes the energy efficiency performance results with respect to number of sensor nodes. 
TABLE 2: Tabulation of energy efficiency.

\begin{tabular}{lccc}
\hline Number of sensor nodes (number) & & Energy efficiency (\%) \\
Opportunistic routing & SRTRBC method \\
\hline 100 & NoC architecture & 90.2 & 96.1 \\
200 & 88.5 & 92.5 & 94.2 \\
300 & 90.6 & 92.6 & 96.5 \\
400 & 89.9 & 93.4 & 97.4 \\
500 & 90.1 & 91.1 & 96.8 \\
600 & 88.6 & 90.2 & 97.8 \\
700 & 88.9 & 91.7 & 95.4 \\
800 & 87.5 & 90.8 & 96.7 \\
900 & 88.1 & 91.5 & 95.3 \\
1000 & 87.2 & 92.2 & 94.1 \\
\hline
\end{tabular}

As shown in Table 2, the three different methods of energy efficiency are SRTRBC method, NoC Architecture [1] and Opportunistic Routing [2]. Among these three different methods, the SRTRBC method outperforms well through increasing the energy efficiency during the data transmission. Let us consider the 100 sensor nodes in wireless network and the initial energy level of every sensor node is predefined as'0.5 J'. Because of transmission process, the energy level of sensor nodes gets reduced. Consequently, the energy efficiency of the SRTRBC method using 100 sensor nodes is $96.1 \%$, while the energy efficiency of the NoC Architecture [1] and Opportunistic Routing [2] is observed as $88.5 \%$ and $90.2 \%$, respectively. Likewise, different energy efficiency results are obtained by applying different number of sensor nodes. The attained energy efficiency results are evaluated for three different existing methods.

Figure 4 illustrates the performance results of energy efficiency linked with three different methods. The $x$-axis represents that the various number of sensor nodes. The $y$-axis denotes the energy efficiency of the sensor nodes. In graphical representation, the blue colour bar denotes the energy efficiency of NoC Architecture [1] and the red colour bar represents the energy efficiency of Opportunistic Routing [2]. The green colour bar symbolizes the energy efficiency of SRTRBC method. The graph denotes that the proposed SRTRBC method outperforms well through increasing the energy efficiency. This is due to the application of softmax regression analysis and Tanimoto reweight boost classification for congestion aware routing in WSN. The route path with lesser buffer space and higher bandwidth capacity is selected as the best route among the multiple paths. The regression analysis identify if the node is energy efficient or nonenergy efficient. The energy-efficient nodes identified the multiple paths from source node to the sink node through transmitting the route request and route reply. This in turn helps to improve the energy efficiency during efficient data transmission in WSN. The average of ten results describes the overall energy efficiency using the proposed SRTRBC method comparatively increased by $9 \%$ and $5 \%$ when compared to other NoC Architecture [1] and Opportunistic Routing [2].

4.2. Impact of Data Delivery Rate. Data delivery rate is computed as ratio of the number of data packets correctly delivered to the total number of data packets sent. The data delivery rate is formulated as

$$
\text { data delivery rate }=\left[\frac{\text { Number of data packet correctly delivered }}{\text { Number of data packets sent }}\right] * 100 \text {. }
$$

From (12), the data delivery rate is determined. The data delivery rate is computed in terms of percentage (\%). Table 3 explains the results of data delivery rate with respect to the number of data packets.

From Table 3, we can conclude that there are three different methods with their data delivery rates: SRTRBC method, NoC Architecture [1], and Opportunistic Routing [2]. Among these three different methods, the SRTRBC method outperforms well through increasing the data delivery rate during the data transmission. Let us consider the 500data packets in wireless network. Consequently, the data delivery rate of the SRTRBC method is $97 \%$, while the data delivery rate of the NoC Architecture [1] and Opportunistic
Routing [2] is observed as $89 \%$ and $92 \%$, respectively. Similarly, different data delivery rate results are attained by applying different number of data packets. The data delivery rate results are determined for three different existing techniques.

Figure 5 explains performance results of data delivery rate for three different techniques. The $x$-axis denotes different number of data packets. The $y$-axis symbolizes the data delivery rate of three different methods. In graphical illustration, the blue colour bar represents the data delivery rate of NoC Architecture [1] and the red colour bar denotes the data delivery rate of Opportunistic Routing [2]. The green colour bar represents the data delivery rate of SRTRBC 


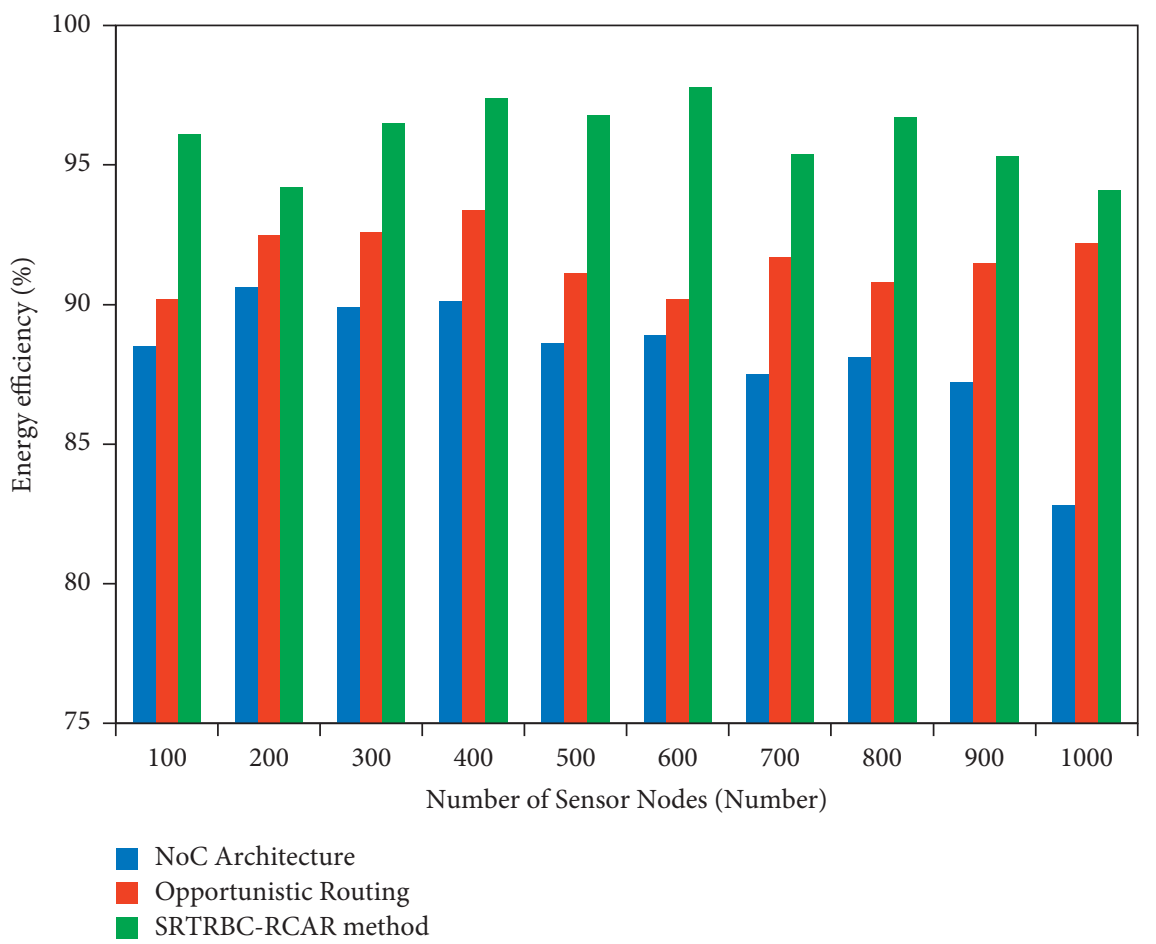

FIgURE 4: Measurement of energy consumption.

TABle 3: Tabulation of data delivery rate.

\begin{tabular}{|c|c|c|c|}
\hline \multirow{2}{*}{ Number of data packets (number) } & \multicolumn{3}{|c|}{ Data delivery rate $(\%)$} \\
\hline & NoC architecture & Opportunistic routing & SRTRBC method \\
\hline 100 & 73 & 80 & 93 \\
\hline 200 & 85 & 88 & 95 \\
\hline 300 & 88 & 90 & 92 \\
\hline 400 & 87 & 88 & 94 \\
\hline 500 & 89 & 92 & 97 \\
\hline 600 & 92 & 94 & 96 \\
\hline 700 & 92 & 95 & 98 \\
\hline 800 & 92 & 95 & 98 \\
\hline 900 & 91 & 96 & 98 \\
\hline 1000 & 95 & 96 & 98 \\
\hline
\end{tabular}

method. The graph represents the proposed SRTRBC method performed well through increasing the data delivery rate. This is due to the application of softmax regression analysis for determining the residual energy level. The regression analysis helps to find if the node is energy efficient or nonenergy efficient. After that, the energy-efficient nodes are recognized and construct the number of paths from source node to the sink node through transmitting route request and route reply. This in turn helps to improve the data delivery rate during efficient data transmission in WSN. The average of ten results explains that the overall data delivery rate using the proposed SRTRBC method is comparatively increased by $5 \%$ and $9 \%$ when compared to other NoC Architecture [1] and Opportunistic Routing [2].
4.3. Impact of Latency. Latency is defined as the variation between the expected arrival time and actual arrival time of data packet. Latency of data transmission is determined as

$$
L=\left[A T_{\text {actual }}\right]-\left[A T_{\text {Expected }}\right] .
$$

From (8), ' $L$ ' denotes the latency. ' $A T_{\text {actual }}$ ' represents the actual arrival time, and ' $A T_{\text {Expected }}$ 'symbolizes the expected arrival time. The latency is computed in milliseconds (ms). Table 4 describes the performance results of latency with respect to the number of data packets varying from 100 to 1000 .

It describes the performance results of latency with respect to the number of data packets varying from 100 to 1000. The table explains latency of three different 


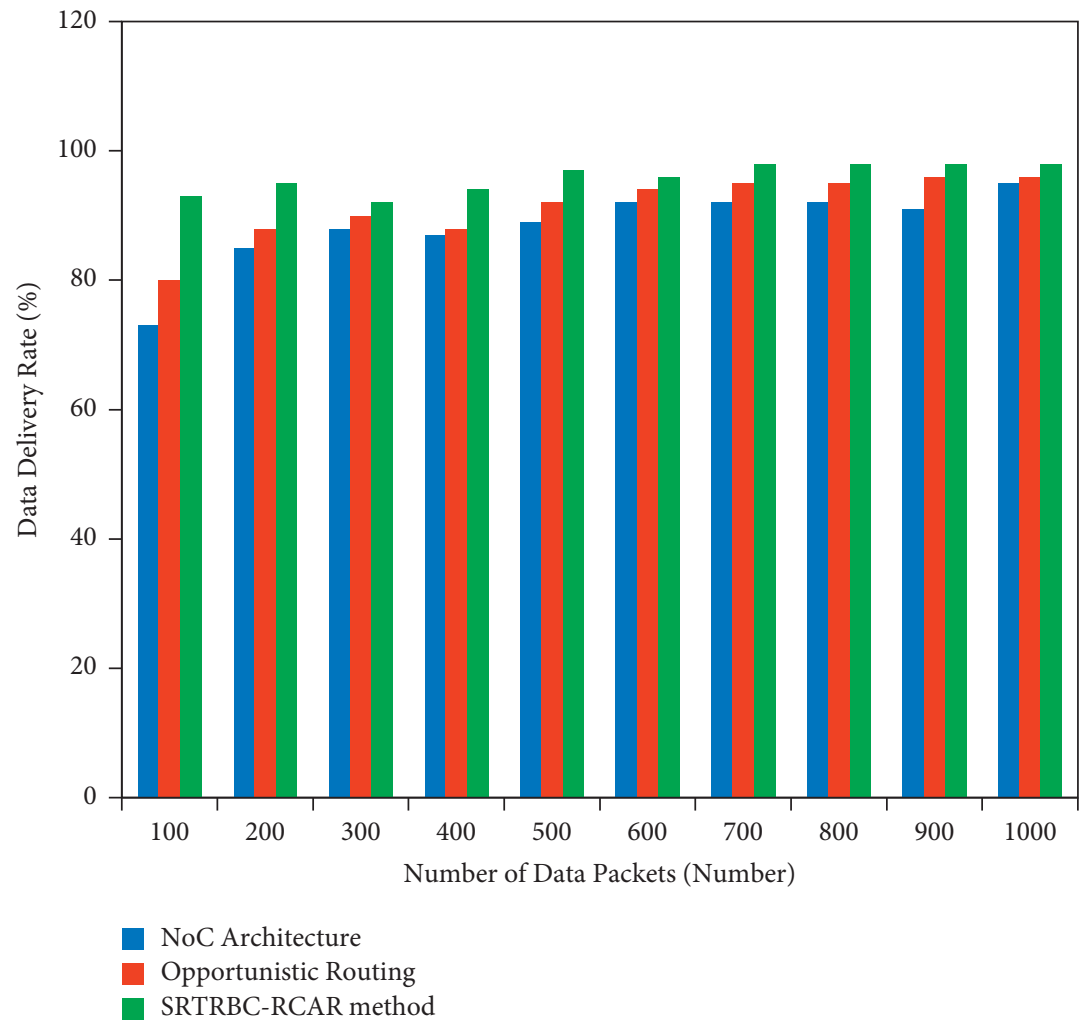

Figure 5: Measurement of data delivery rate.

TABLE 4: Tabulation of latency.

\begin{tabular}{|c|c|c|c|}
\hline \multirow{2}{*}{ Number of data packets (number) } & \multicolumn{3}{|c|}{ Latency (ms) } \\
\hline & NoC architecture & Opportunistic routing & SRTRBC method \\
\hline 100 & 31 & 22 & 15 \\
\hline 200 & 33 & 26 & 19 \\
\hline 300 & 37 & 29 & 21 \\
\hline 400 & 40 & 32 & 25 \\
\hline 500 & 43 & 35 & 27 \\
\hline 600 & 47 & 38 & 30 \\
\hline 700 & 50 & 41 & 32 \\
\hline 800 & 52 & 44 & 35 \\
\hline 900 & 55 & 47 & 37 \\
\hline 1000 & 57 & 49 & 40 \\
\hline
\end{tabular}

methods, SRTRBC method, NoC Architecture [1], and Opportunistic Routing [2]. Among three different methods, the SRTRBC method attained better results through reducing the latency during the data transmission. Let us consider the number of data packets is 800 in wireless network. Consequently, the latency of the SRTRBC method is $35 \mathrm{~ms}$ while the latency of the NoC Architecture [1] and Opportunistic Routing [2] is observed as $52 \mathrm{~ms}$ and $44 \mathrm{~ms}$, respectively. Similarly, different latency performance results are attained for different number of data packets. The latency results are computed for three different existing techniques.

Figure 6 explains the performance results of latency for three different methods. The $x$-axis denotes different number of data packets. The $y$-axis symbolizes the latency of the three different methods. In graphical illustration, the blue colour bar represents the latency of NoC Architecture [1] and the red colour bar denotes latency of Opportunistic Routing [2]. The green colour bar symbolizes the latency of SRTRBC method. The graph represents the proposed 


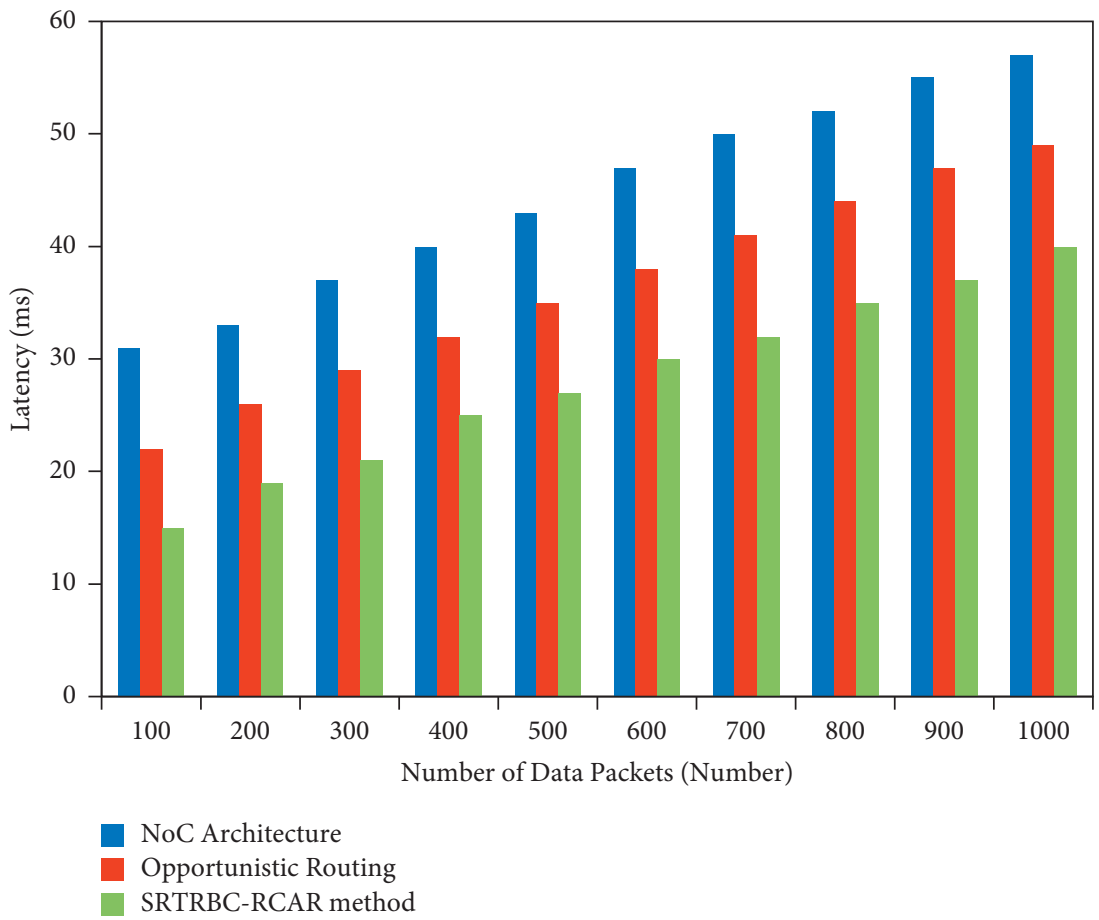

FIgURE 6: Measurement of latency.

TABLE 5: Tabulation of data loss rates.

\begin{tabular}{lccc}
\hline Number of data packets (number) & Data loss rate (\%) \\
Opportunistic routing & SRTRBC method \\
\hline 100 & NoC architecture & 20 & 7 \\
200 & 15 & 12 & 5 \\
300 & 12 & 10 & 8 \\
400 & 13 & 8 & 6 \\
500 & 11 & 6 & 4 \\
600 & 8 & 5 & 2 \\
700 & 8 & 5 & 2 \\
800 & 8 & 4 & 2 \\
900 & 9 & 4 & 2 \\
1000 & 5 & 4 & \\
\hline
\end{tabular}

SRTRBC method performs well through reducing the latency. This is because of applying the softmax regression analysis for calculating the residual energy level. The regression analysis helps to find if the node is energy efficient or non-energy efficient. The energy-efficient nodes are identified and construct multiple paths from source node to destination through sending the route request message and route reply message. This in turn helps to minimize the latency during efficient data transmission in WSN. The average of ten results explains that the overall latency using the proposed SRTRBC method is comparatively reduced by
$38 \%$ and $23 \%$ when compared to other NoC Architecture [1] and Opportunistic Routing [2].

4.4. Impact of Data Loss Rate. Data loss rate is computed as the ratio of the number of data packets correctly delivered to the total number of data packets transmitted. The data delivery rate is formulated as

data loss rate $=\left[\frac{\text { Number of data packet lost }}{\text { Number of data packets sent }}\right] * 100$. 


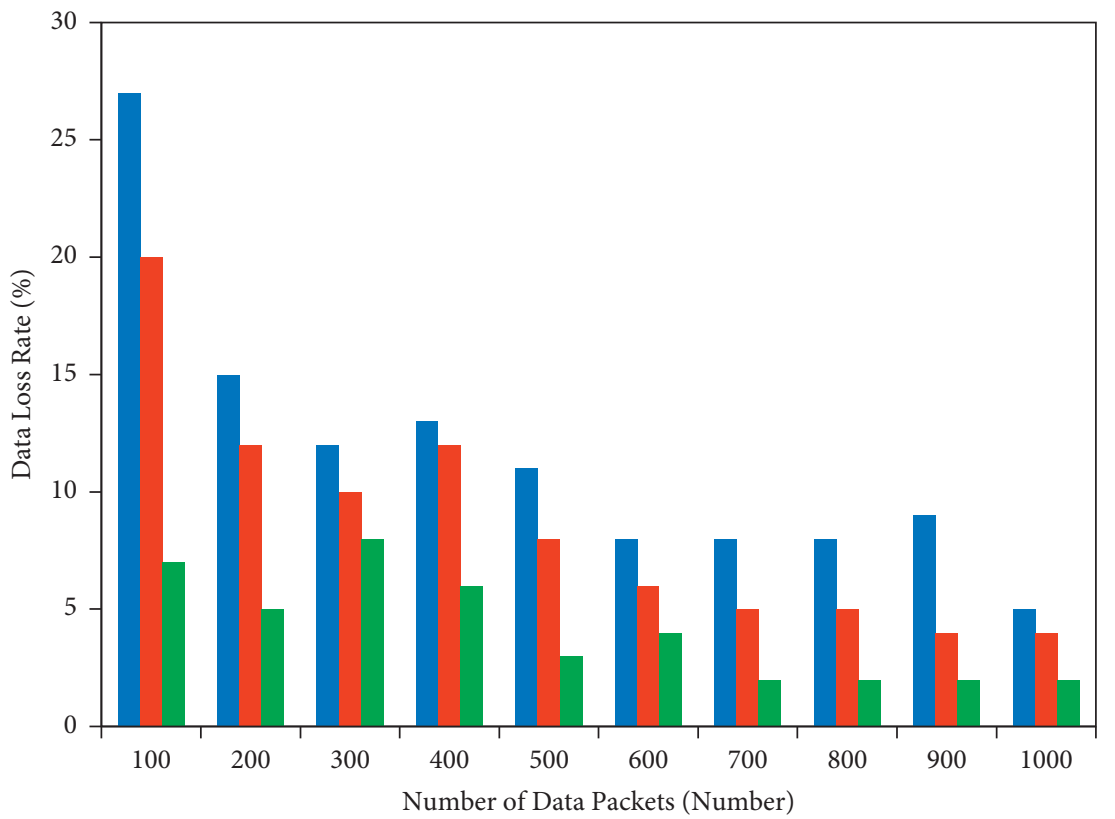

NoC Architecture

Opportunistic Routing

SRTRBC-RCAR method

Figure 7: Measurement of data loss rate.

TABLE 6: Tabulation of throughput.

\begin{tabular}{lccc}
\hline Number of sensor nodes (number) & \multicolumn{2}{c}{$\begin{array}{c}\text { Throughput (bps) } \\
\text { Opportunistic routing }\end{array}$} & \begin{tabular}{l} 
SRTRBC method \\
\hline 100
\end{tabular} \\
200 & 160 & 209 & 184 \\
300 & 181 & 301 & 245 \\
400 & 265 & 395 & 327 \\
500 & 367 & 457 & 406 \\
600 & 403 & 506 & 498 \\
700 & 491 & 649 & 587 \\
800 & 619 & 767 & 681 \\
900 & 726 & 865 & 804 \\
1000 & 811 & 954 & 887 \\
\hline
\end{tabular}

From (14), the data loss rate is determined. The data loss rate is measured in terms of percentage (\%). Table 5explains the performance results of data loss rate with respect to the number of data packets.

In Table 5, the data loss rate of three different methods (SRTRBC, NoC Architecture [1], and Opportunistic Routing [2]) is given. Among these three different methods, the SRTRBC method reduces the data loss rate during the data transmission. Let us consider the 900 data packets in wireless network. Consequently, the data loss rate of the SRTRBC method is $2 \%$ while the data loss rate of the NoC Architecture [1] and Opportunistic Routing [2] is observed as $9 \%$ and ,4\% respectively. Similarly, different data loss rate results are obtained by applying different number of data packets. The data loss rate results are computed for three different existing techniques.
Figure 7 explains the performance results of data loss rate for three different techniques. The $x$-axis denotes different number of data packets. The $y$-axis represents the data loss rate of the three different methods. In graphical illustration, the blue colour bar denotes the data loss rate of NoC Architecture [1] and the red colour bar denotes the data loss rate of Opportunistic Routing [2]. The green colour bar represents the data loss rate of SRTRBC method. The graph represents the proposed SRTRBC method performed well through reducing the data loss rate. This is due to the application of softmax regression analysis for computing the residual energy level. The regression analysis helps to identify if the node is energy efficient or nonenergy efficient. With energyefficient nodes, multiple paths are constructed from source node to the sink node through transmitting the 


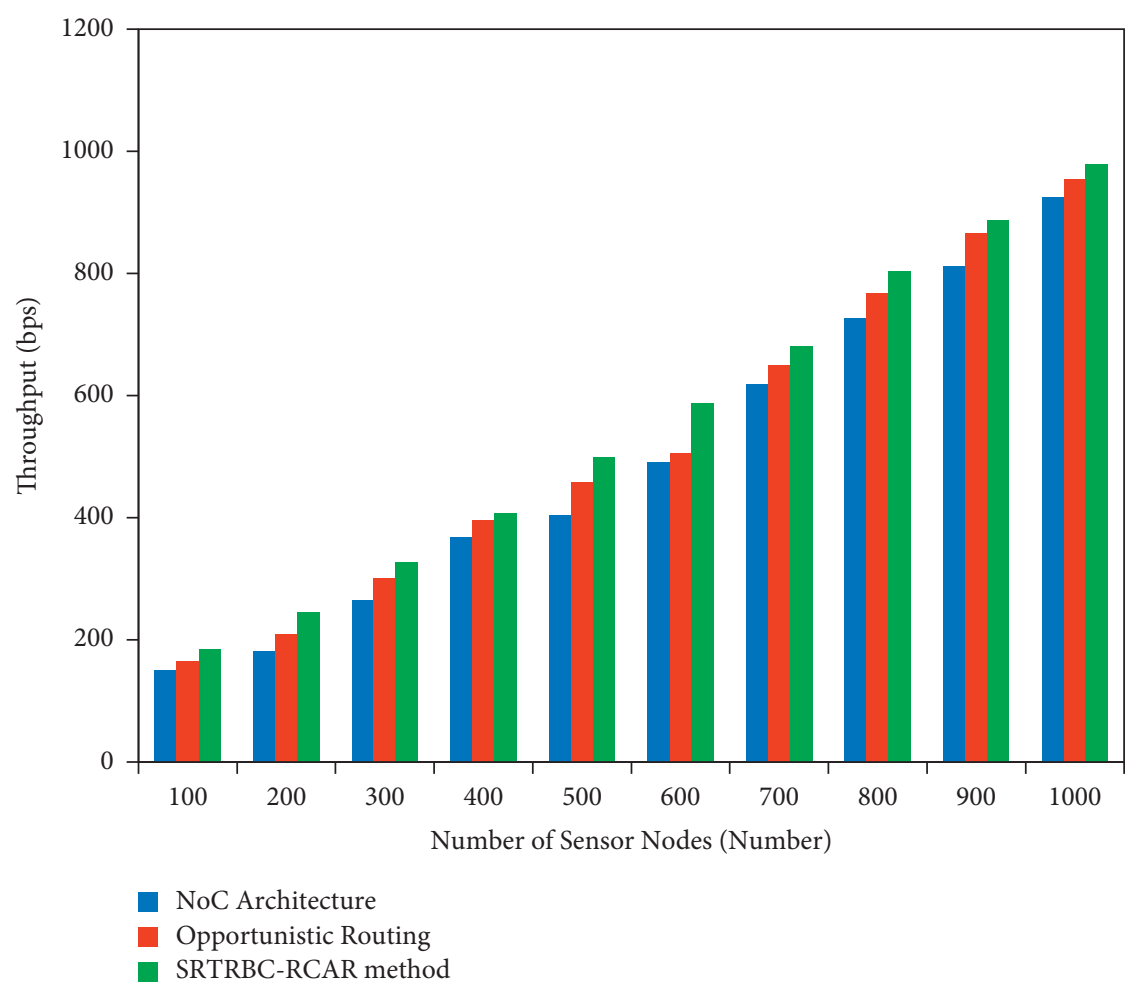

FIGURE 8: Measurement of throughput.

route request and route reply. This in turn helps to reduce the data loss rate during efficient data transmission in WSN. The average of ten results explains that the overall data loss rate using proposed SRTRBC method is comparatively reduced by $64 \%$ and $51 \%$ when compared to other NoC Architecture [1] and Opportunistic Routing [2].

4.5. Impact of Throughput. Throughput is defined as the amount of packets broadcasted from the sensor nodes at a certain interval. It is measured as

$$
T=\frac{\text { number of packets transmitted }}{\text { time interval }} .
$$

From (15), the throughput ' $T$ ' is determined. Throughput is measured in bits per seconds (bps). Table 6 describes the performance result of throughput with respect to the number of data packets.

It portrays the throughput of three different methods, SRTRBC method, NoC Architecture [1], and Opportunistic Routing [2]. We see that the SRTRBC method increases the throughput during the data transmission. Let us consider the 800 data packets in wireless network. Consequently, the throughput of the SRTRBC method is 804 bps, while the throughput of the NoC Architecture [1] and Opportunistic Routing [2] is observed as 726 bps and 767 bps, respectively. Similarly, different throughput results are obtained for different number of sensor nodes. The throughput results are computed for three different existing techniques.
Figure 8 explains the performance results of throughput for three different techniques. The $x$-axis denotes different number of sensor nodes. The $y$-axis denotes the throughput of the three different methods. In graphical illustration, the blue colour bar represents the throughput of NoC Architecture [1] and the red colour bar denotes the throughput of Opportunistic Routing [2]. The green colour bar represents the throughput of SRTRBC method. The graph represents the proposed SRTRBC method performed well through increasing the throughput. This is because of applying softmax regression analysis and Tanimoto reweight boost classification for congestion aware routing depending on buffer space and bandwidth capacity. The regression analysis identify if the node is energy efficient or nonenergy efficient. With energy-efficient nodes, multiple paths are constructed from source node to the sink node through transmitting the route request and route reply. In this way, it gets increased during efficient data transmission in WSN. The average of ten results explains that the overall throughput using proposed SRTRBC method is comparatively increased by $17 \%$ and $8 \%$ when compared to other NoC Architecture [1] and Opportunistic Routing [2].

\section{Conclusion}

A new routing method termed SRTRBC method is introduced to route packets around congested areas through identifying the energy-efficient and underloaded nodes in WSN. SRTRBC method comprised route path construction and congestion aware MIMO routing. The node residual 
energy is determined for route path construction in WSN. Softmax regression analysis examines the residual energy level to identify if the node is energy efficient or nonenergy efficient. The energy-efficient nodes constructed multiple paths from source node to the sink node through route request and route reply. The Tanimoto Reweight Boost Classification method performed the congestion aware routing with minimal latency and packet loss during the data transmission in WSN. The qualitative and quantitative results discussion shows that SRTRBC method attained better performance in terms of achieving higher data delivery rate and lesser latency and energy efficiency when compared to other related works. The future direction of the work can be carried out through introducing the deep learning concept during MIMO routing in WSN. With the help of deep learning method, the energy efficiency gets increased at the required level and latency time gets reduced.

\section{Data Availability}

The data used to support the findings of this study are available from the corresponding author upon request.

\section{Conflicts of Interest}

The authors declare that there are no conflicts of interest.

\section{Acknowledgments}

This research was supported by the Researchers Supporting Project number (RSP-2021/244), King Saud University, Riyadh, Saudi Arabia.

\section{References}

[1] M. Devanathan, V. Ranganathan, and P. Sivakumar, "Congestion-aware wireless network-on-chip for high-speed communication," Journal for Control, Measurement, Electronics, Computing and Communications, vol. 61, no. 1, pp. 92-98, 2020.

[2] Y. Richter and I. Bergel, "Opportunistic routing based on partial CSI in MIMO random ad-hoc networks," EURASIP Journal on Wireless Communications and Networking, vol. 2021, no. 131, pp. 1-15, 2021.

[3] N. Taherkhani, R. Akbar, F. Safaei, and M. Moudi, "A congestion-aware routing algorithm for mesh-based platform networks-on-chip," Microelectronics Journal, Elsevier, vol. 114, no. 1, pp. 1-14, 2021.

[4] A. Nitanda and T. Suzuki, "Functional gradient boosting for learning residual-like networks with statistical guarantees," Proceedings of Twenty Third International Conference on Artificial Intelligence and Statistics, vol. 108, no. 1, pp. 1-10, 2020.

[5] Q. Miao, Y. Cao, X. Ge, M. Gong, J. Liu, and J. Song, "RBoost: label noise-robust boosting algorithm based on a nonconvex loss function and the numerically stable base learners," IEEE Transactions on Neural Networks and Learning Systems, vol. 27, no. 11, pp. 2216-2228, November 2016.

[6] K. Ahmad, M. A. J. Sethi, R. Ullah et al., "Congestion-aware routing algorithm for NoC using data packets," Wireless Communications and Mobile Computing, vol. 2021, Article ID 8588646, 11 pages, 2021.
[7] G. Sangeetha, M. Vijayalakshmi, S. Ganapathy, and A. Kannan, "An improved congestion-aware routing mechanism in sensor networks using fuzzy rule sets," Peer-to-Peer Networking and Applications, Spring, vol. 13, no. 1, pp. 890-904, 2020.

[8] P. Aimtongkham, P. Horkaew, and C. So-In, "Multistage fuzzy logic congestion-aware routing using dual-stage notification and the relative barring distance in wireless sensor networks," Wireless Networksg, vol. 27, no. 1, pp. 1287-1308, 2021.

[9] H. S. Das and S. Bhattacharjee, "A congestion aware routing for lifetime improving in grid-based sensor networks," Journal of High Speed Networks, vol. 23, no. 1, pp. 1-14, 2017.

[10] M. Farsi, M. Badawy, M. Moustafa, H. A. Arafat, and Y. Abdulazeem, "A congestion-aware clustering and routing (CCR) protocol for mitigating congestion in WSN," IEEE Access, vol. 7, no. Issue 1, pp. 105402-105419, 2019.

[11] K. Singh, K. Singh, L. H. Son, and A. Aziz, "Congestion control in wireless sensor networks by hybrid multi-objective optimization algorithm," Computer Networks, Computer Networks, vol. 138, no. 6, pp. 90-107, 2018.

[12] B. Sharma, G. Srivastava, and J. C.-W. Lin, "A bidirectional congestion control transport protocol for the internet of drones," Computer Communications, Elsevier, vol. 153, no. 3, pp. 102-116, 2020.

[13] L. J. YaoYukun, R. Z. XuDongliang, and H. U. Qing, "Centralized congestion control routing protocol based on multimetrics for low power and lossy networks," The Journal of China Universities of Posts and Telecommunications, Elsevier, vol. 24, no. 5, pp. 35-43, 2017.

[14] C. Suwannapong and C. Khunboa, "EnCoCo-RED.: enhanced congestion control mechanism for CoAP observes group communication," Ad Hoc Networks, Elsevier, vol. 112, no. 3, pp. 1-12, 2021.

[15] S. Qu, L. Zhao, and Z. Xiong, "Cross-layer congestion control of wireless sensor networks based on fuzzy sliding mode control," Neural Computing and Applications, Spring, vol. 32, no. 3, pp. 13505-13520, 2020.

[16] V. Srivastava, S. Tripathi, K. Singh, and L. H. Son, "Energy efficient optimized rate based congestion control routing in wireless sensor network," Journal of Ambient Intelligence and Humanized Computing, Springer, vol. 11, no. 1, pp. 1325-1338, 2020.

[17] S. L. Yadav, R. L. Ujjwal, S. Kumar, O. Kaiwartya, M. Kumar, and P. K. Kashyap, "Traffic and energy aware optimization for congestion control in next generation wireless sensor networks," Journal of Sensors, Hindawi Publishing Corporation, vol. 2021, Article ID 5575802, 16 pages, 2021.

[18] M. Hossein Homaei, F. Soleimani, S. Shamshirband, A. Mosavi, N. Nabipour, and A. R. Varkonyi-koczy, "An enhanced distributed congestion ControlMethod for classical 6LowPAN ProtocolsUsing fuzzy decision system," IEEE Access, vol. 8, no. 1, pp. 20628-20645, 2020.

[19] D. Waghole, V. Deshpande, D. Midhunchakkaravarthy, and M. Jadhav, "Position aware congestion control (PACC) algorithm for disaster management system using WSN to improve QoS," Design Engineering, vol. 2021, no. 7, pp. 11470-11478, 2021.

[20] M. Masdari, "Energy efficient clustering and congestion control in WSNs with mobile sinks," Wireless Personal Communications, Springer, vol. 111, no. 1, pp. 611-642, 2020. 